\title{
NBC Model for Early Prediction of At-Risk Students in Course
}

\author{
P. Sunanda, D. Kavitha
}

\begin{abstract}
Increase in computer usage for different purposes in different fields has made the computer important to learn things. Machine learning made systems to learn things and work accordingly on their own. Among the different fields that use machine learning, the education field is one. In the education field, machine learning has led to the advent of a digital-enabled classroom, speech recognition, adaptive learning techniques, and development of artificial instructor. Along with this, the prediction has its importance. In the education field, the main problem is students drop out. The machine learning predictive modeling approach can be used to identify the students who are at-risk and inform the instructor and students before reducing the dropouts. The main intention of this paper is to model a system that could be a solution to reduce the drop-outs and increase the education standards in students by early predicting their risk in a course.
\end{abstract}

Keywords: Machine Learning, Prediction, at-risk, Naive-Bayes.

\section{INTRODUCTION}

The world and the society around us say the importance of education through the inventions of new things each second. Education has become the basic need for a human to survive in this updated world. In this century, every child has many resources to acquire education. One such and most older resource is school or college which is an offline interactive mode of educating a child either using a student-centered approach or a teacher-centered approach. In either of these, a student can be guaranteed to gain knowledge. The problem here is since everyone is not equally intelligent, based on their understanding level, listening skills, attending the classes, interest in the course, there will be an effect in percentages or marks of the students. This results in dropouts in most schools and colleges.

This paper focuses mainly to predict different factors that affect student education. It predicts based on the marks, analyzing power of the student and then notifies him if he/she falls under risk or not before completing the course.

Revised Manuscript Received on February 05, 2020.

* Correspondence Author

P. Sunanda, Assistant Professor, Department of Computer Science \& Engineering, G. Pulla Reddy Engineering College, Kurnool India.

Dr. D. Kavitha, Professor, Department of Computer Science \& Engineering at G. Pulla Reddy Engineering College, Kurnool India.

(c) The Authors. Published by Blue Eyes Intelligence Engineering and Sciences Publication (BEIESP). This is an open access article under the CC BY-NC-ND license (http://creativecommons.org/licenses/by-nc-nd/4.0/)

\section{MACHINE LEARNING}

\subsection{Introduction to Machine Learning}

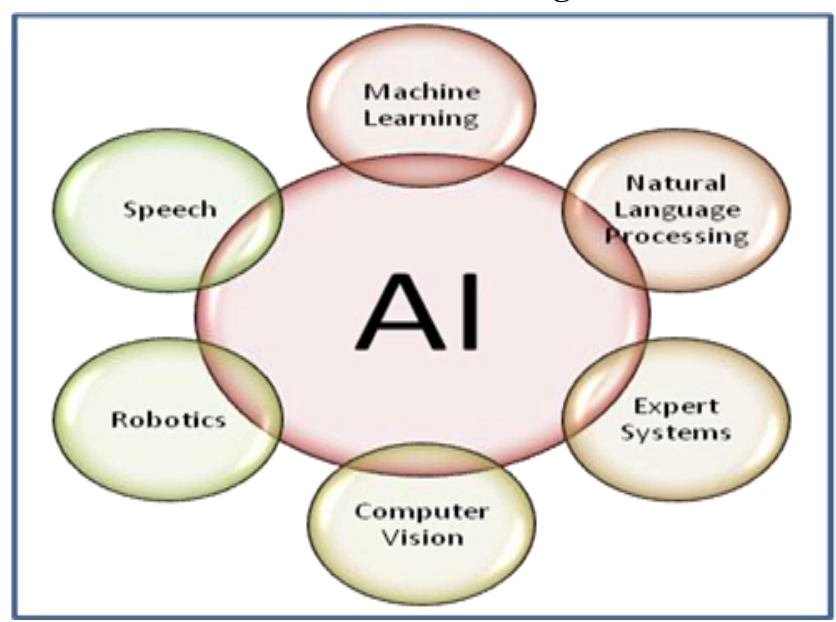

Figure1. Artificial Intelligence with its fields

Artificial Intelligence (AI) is the ability of a computer or a machine to think and learn. It has many fields like Natural Language processing, expert systems, robotics, etc. to make the computers smart. Among these, Machine Learning is one that allows computers or machines to automatically learn from past data, to enable data-driven design without any explicit programming.

In the year 1959, Arthur Samuel coined the name Machine Learning which explains the study and the construction of algorithms. These algorithms are also designed in such a way that, they can learn and improve themselves when exposed to the new data.

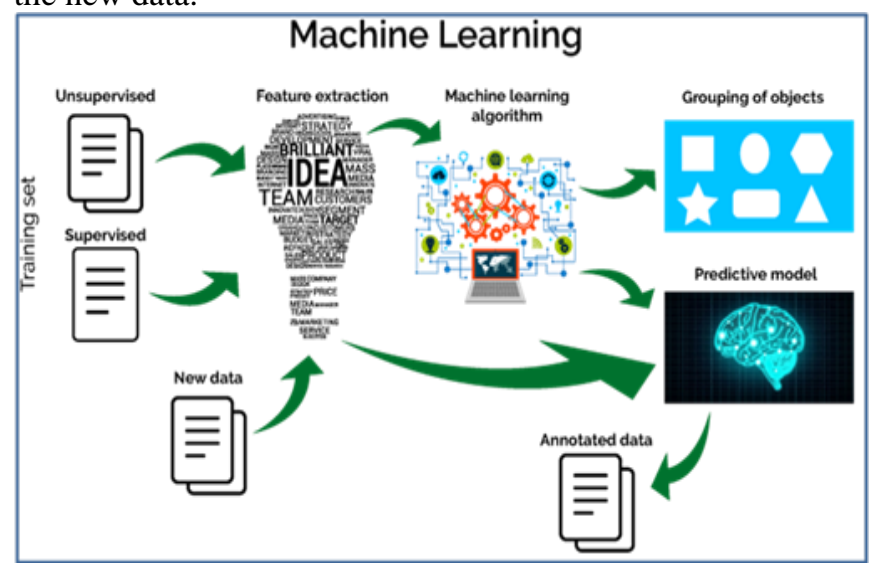

Figure2. Machine learning process

Published By:

Blue Eyes Intelligence Engineering

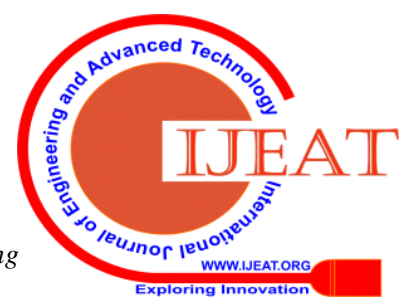




\section{NBC Model for Early Prediction of At-Risk Students in Course}

\subsection{Classification of Machine Learning tasks}

Machine learning tasks are classified typically into several broad categories as follows

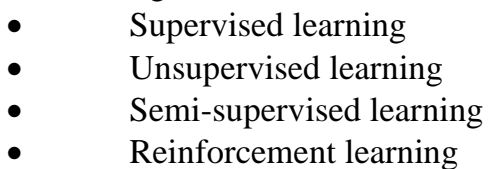

\section{- Reinforcement learning}

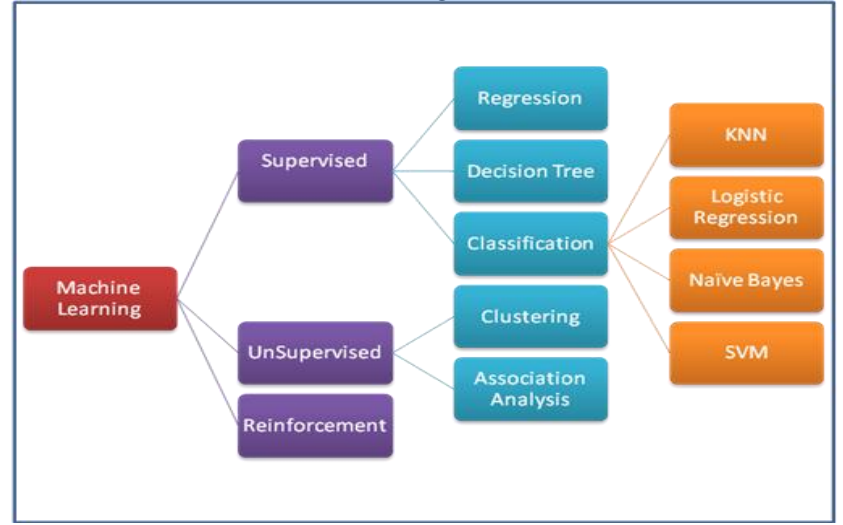

Figure3. Classification of Machine Learning

\section{1) Supervised Learning}

It is a task in which based on the example input-output pair called labelled dataset, the learning function maps input to an output. In this task, the model will get trained on the labelled dataset. The applications of supervised learning are

- Handwriting recognition

- Spam detection

- $\quad$ Pattern recognition

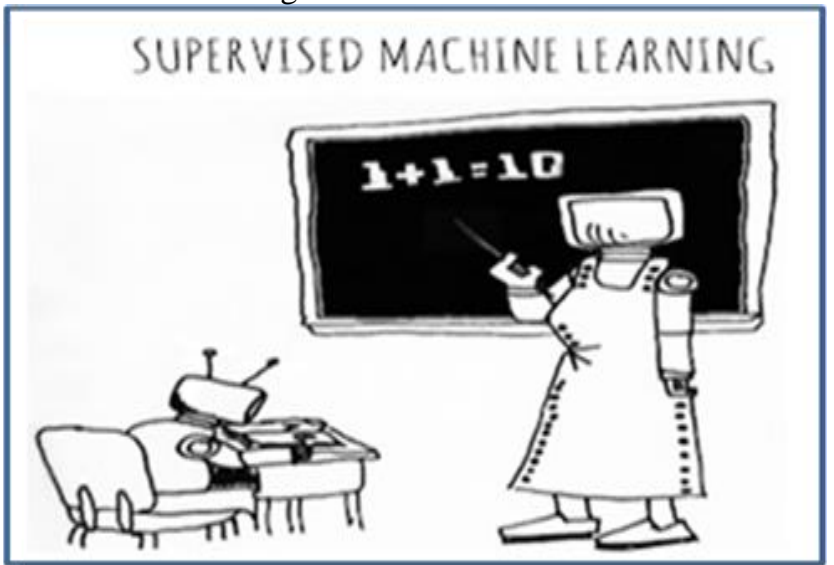

Figure4. Supervised Learning

\section{2) Unsupervised Learning}

This task helps in finding the unknown dataset patterns without pre-existing labels. It mainly deals with unlabelled data. The applications of unsupervised learning are

- Density estimation in statistics

- Clustering

- Dimensionality Reduction

- Visualization

- Anomaly Detection

\section{3) Semi-Supervised Learning}

This task combines a smaller volume of labelled data with larger volumes of unlabelled data. So, it falls in between supervised and unsupervised learning. The applications of semi-supervised learning are

- Speech analysis
- Web content classification

- Protein sequence classification

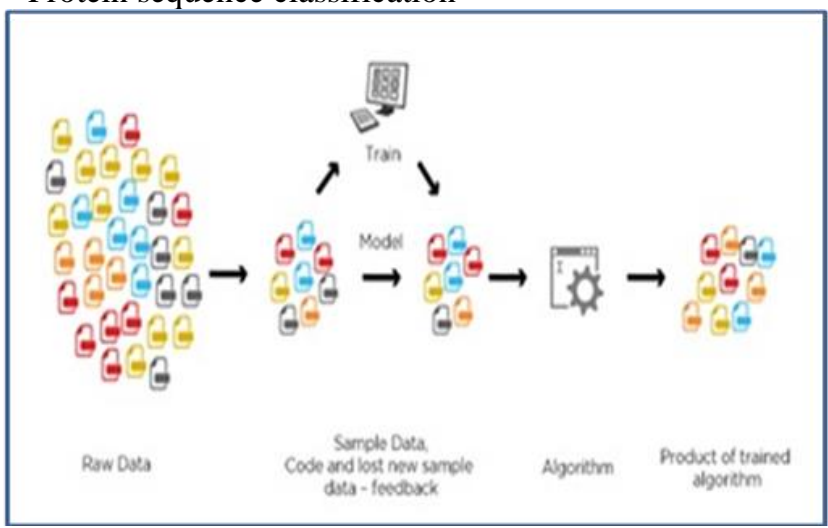

Figure5. Working model of Semi-Supervised Learning

\section{4) Reinforcement Learning}

It is a type of dynamic programming that trains algorithms to deal with taking suitable action to maximize the reward of a particular situation. The applications of Reinforcement Learning are

- $\quad$ Traffic Light Control

- $\quad$ Bidding and Advertising

- Web System Configuration

\subsection{How does Machine Learning Works?}

Machine learning has 7 major steps they are as follows

Step 1: Gathering Data - Predict model accuracy directly proportional to the quality of data.

Step 2: Data preparation - Prepare data as per our model and remove unnecessary data.

Step 3: Choosing a model - The most typical job is to select the most suitable model.

Step 4: Training - Train the model according to the dataset

Step 5: Evaluation - Evaluate Training dataset.

Step 6: Parameter Tuning - After evaluation, important parameters can be identified and added

Step 7: Prediction - And the final step is predicting using the model and dataset.

The working model of Machine Learning can be shown in the following figure.

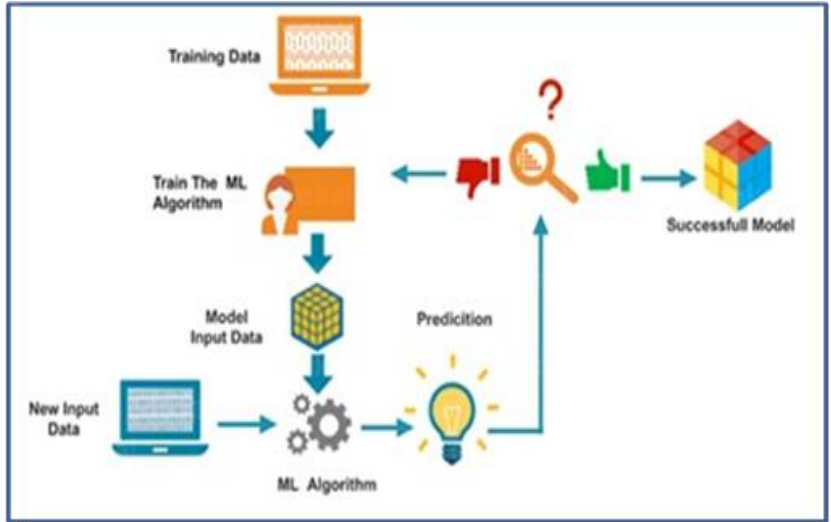

Figure6. Working Model of Machine Learning

Published By:

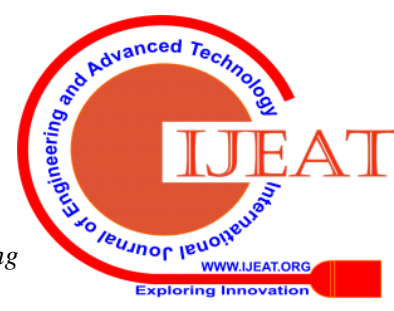




\subsection{Applications of Machine Learning}

- Email Spam and Malware Filtering

- Search Engine Result Refining

- Playing Video Games Automatically

- Education

- Finance [1].

\section{TECHNOLOGIES USED}

To predict student's risk factors using Machine learning, a programming language with machine learning libraries is needed.

\subsection{Available Languages}

There are many programming languages available like C, Java, R, Python. Of these languages, every language has its' own specific role to be used to implement a particular technology. So, to implement machine learning algorithms and to work with a complex set of tasks, the preferred programming language is python.

\subsection{Why Python?}

According to the survey of Stacks Overflow in the year 2019, Python has raised in the ranks to the 4th position from among the most commonly used programming languages, from the 7th position in 2018. It is the fastest-growing major programming language today. It is the second most loved language.

There are several reasons like simple syntax, easy programming, many built-in modules, libraries, functions and methods that made to select python. Among built-in modules, the sklearn (scikit-learn) called "Machine Learning in Python" is used to work with machine learning algorithms to predict at-risk students.

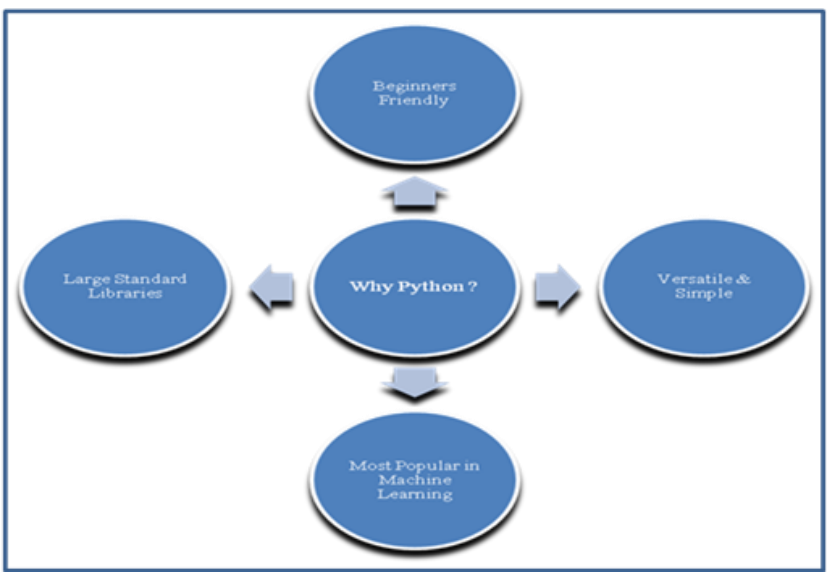

Figure7. Why Python?

\subsection{Packages and Libraries in Python}

The following table Tabe1 lists a set of built-in modules in python to work with machine learning algorithms

Table1. Python built-in modules

\begin{tabular}{|l|l|}
\hline Module & Description \\
\hline numpy & $\begin{array}{l}\text { To work with N-dimensional } \\
\text { array objects }\end{array}$ \\
\hline pandas & $\begin{array}{l}\text { To analyze the data through data } \\
\text { frames }\end{array}$ \\
\hline matplotlib & To create 2D graphs and plots \\
\hline
\end{tabular}

\begin{tabular}{|l|l|}
\hline scikit-learn & $\begin{array}{l}\text { To work with machine learning } \\
\text { algorithms }\end{array}$ \\
\hline seaborn & To visualize the data \\
\hline
\end{tabular}

\section{PROPOSED PREDICTION MODELS}

The six prediction models are

- Logistic Regression (Log Reg)

- Support Vector Machine(SVM)

- Decision Tree (DT)

- Multi-Layer Perceptron (MLP)

- Naïve Bayes Classifier

- K- Nearest Neighbor (KNN)

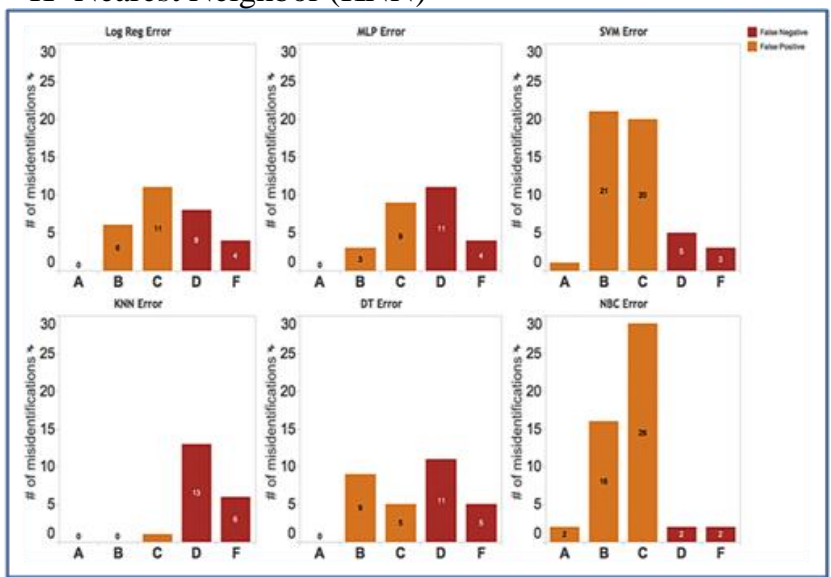

Figure8. Graphs showing the number of misidentifications

- Logistic Regression (Log Reg) in general is used to model the probability of certain existing events and classes such as pass/fail, win/lose, yes/no, more/less. In the same way, here it can be used to classify the students based on pass/fail, interested/uninterested, risk/no risk.

- Support Vector Machine (SVM) is a supervised learning model used for classification and regression associated algorithms. These associated algorithms are used to analyze the data. The analysis can be done through a kernel trick technique by transforming the data. And based on these data transformations, an optimal boundary between possible outputs will be found by it. Here the students can be classified based on their gender, test preparation course, parental education level.

- Decision Tree (DT) is a partitioning method model. Its structure looks like a tree with decisions and consequences. The consequences are selected based on the calculated entropy value of the decision. The fate of the consequence mainly depends on the entropy of the decision. Here the consequences like student interest in a course, predicting his risk factor mainly depends on the decisions of math score, reading and writing scores.

- Multi-Layer Perceptron (MLP) is an Artificial Neural Network (ANN) with one input, an arbitrary number of hidden layers and one output layer.

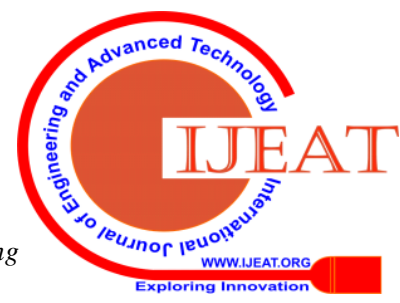




\section{NBC Model for Early Prediction of At-Risk Students in Course}

The input layer receives the input, the input is processed or computed by the hidden layers and decision or prediction is performed by the output layer. So, the input and output layers are fixed but the hidden layer count changes based on several computations to be performed. Here the student gender or parental education level can be taken as the input layer, the math, reading and writing scores are to be treated as hidden layers, and predicting the risk factor of a student is done by output layer.

- Naive Bayes Classifier (NBC) is a simple probabilistic classifier family member that follows the "Bayes theorem". It follows Naïve independence assumptions between features. This is a family of algorithms that share "every classifier feature is equally important and independent of each other. Here the features such as gender, parental educational level, test preparation course, math score, reading score, and writing score are considered. The gender and parental educational level are independent of one another and race/ethnicity is independent of reading, writing and math scores. Even though these features are independent of one another, but they have their importance in predicting the student risk.

- K-Nearest Neighbor (KNN) is a simple, non-parametric algorithm used for both classification and regression. It stores all available cases and classifies new cases based on the similarity measures. It's being in use for statistical estimation and pattern recognition since the 1970s. Here, based on student reading, writing, and math score, they can be classified as two sets one set with a set of students whose score is less than 50 and other greater than 50 . The students in set less than 50 will be almost at risk. So, it becomes easy for prediction.

\section{DATA PRE-PROCESSING AND EXPLORATORY ANALYSIS}

Here all the steps needed for prediction like from data collection, data pre-processing, modelling are followed.

\subsection{Data Source}

Data is the primary thing needed to perform any operation. A data source is like a repository of data in different formats. It contains data as hard-coded data, datasets, spreadsheets, etc. Here the students performance dataset with 1000 records sis taken from 4Shared.com data source.

This dataset has many features like gender, race/ethnicity, and parental level of education, lunch, and test preparation course, math score, reading score and writing score. The Figure9. gives a snapshot of the dataset.

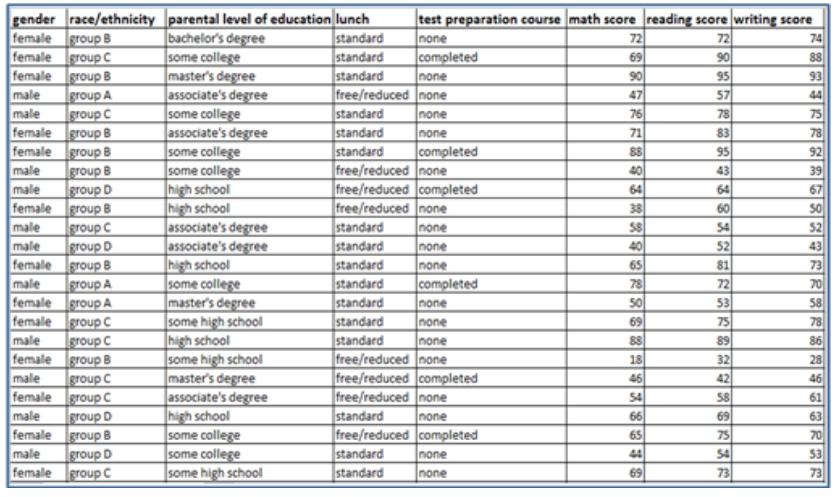

Figure9. Snapshot of the dataset

\subsection{Dataset Description}

- Gender: In developing countries like India, gender inequality plays a main role in education. So, here this feature is considered to check the effect of it on student's studies. Here Male and Female are two genders considered.

- Race: The race/ethnicity in the data set says to which group of race the student belongs. Based on the student race, it becomes easy to predict his risk factor.

- Parental level Education: The parental level education in the dataset about the degree of the parent.

- Test preparation course: The test preparation course in the dataset describes the course details of the student whether he has taken/completed the course or not.

- Math score: Math score in the dataset describes the score scored by the student.

- Reading score: The score that is scored by the student in the general daily basis reading tests.

- Writing score: The score that is score by the student in the general daily basis writing tests.

\subsection{Data Pre-Processing}

Before pre-processing the data it must be loaded into Data Frames.

\section{\#Reading data into python data frames}

data=pd.read_csv("C:/input/studentsperformance/StudentsPerformance.csv") data.head()

\begin{tabular}{|c|c|c|c|c|c|c|c|c|}
\hline \multicolumn{8}{|c|}{ I deta. Dataframe } & $-\square x$ \\
\hline Inder & gender & reselethicty & intel erel of educi & lunch & iptepertion cou & meth sole & readingscolt & writing score ^ \\
\hline$\theta$ & fenale & grove B & $\begin{array}{l}\text { bachelor's } \\
\text { degree }\end{array}$ & standard & none & 72 & 72 & 74 \\
\hline 1 & fenale & group $C$ & sone college & standard & completed & 69 & 98 & 88 \\
\hline 2 & ferale & group B & $\begin{array}{l}\text { naster's } \\
\text { degree }\end{array}$ & standard & none & 90 & 95 & 93 \\
\hline 3 & nale & group A & $\begin{array}{l}\text { associate's } \\
\text { degree }\end{array}$ & frree/reduced & none & 47 & 57 & 44 \\
\hline 4 & male & group 6 & sore college & standard & none & 76 & 78 & 75 \\
\hline 5 & ferale & grove $B$ & $\begin{array}{l}\text { associate's } \\
\text { deegree }\end{array}$ & standard & none & 71 & 83 & 78 \\
\hline 6 & ferale & group B & sone college & standard & conpleted & 88 & 95 & 92 \\
\hline 7 & nale & grove B & sone college & frree/reduced & none & 48 & 43 & 39 \\
\hline 8 & nale & group 0 & high school & ifree/reduced & completed & 64 & 64 & 67 \\
\hline 9 & fenale & group B & highn school & freetreduced & none & 38 & 68 & 58 \\
\hline 10 & nale & group 6 & $\begin{array}{l}\text { associate's } \\
\text { degree }\end{array}$ & standard & none & 58 & 54 & 52 \\
\hline Femot & Reses $\quad \nabla_{B}$ & 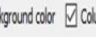 & Cidm minmax & & & & & Coned \\
\hline
\end{tabular}

Figure10. Output after reading dataset into python

After successfully importing the dataset as shown in the Figure10, it is observed that the column names of the dataset seem to be confusing. So, they need to be renamed for better understanding and easy deploy and debug. The dataset with renamed columns is shown in Figure11. 


\section{1) Renaming columns:}

\#Renaming columns

data.columns=['gender','race','parentsdegree','lunch','course','mathscore','readings core','writingscore']

data.head()

\begin{tabular}{|c|c|c|c|c|c|c|c|c|}
\hline & gendel & laCe & parentisdegeree & lunch & course & mathscore & readingscore & witingscore \\
\hline 0 & female & group $B$ & bachelor's degree & standard & none & 72 & 72 & 74 \\
\hline 1 & lemale & group C & some college & standaro & completed & 69 & 90 & 88 \\
\hline ? & female & group B & master's deogete & siandarto & none & 90 & 95 & 93 \\
\hline 3 & male & grouph & assoodate's degyee & treelreduced & none & 47 & 57 & 44 \\
\hline 4 & male & group C & some college & siandaro & none & 76 & 78 & 75 \\
\hline
\end{tabular}

Figure11. Data frame after renaming the columns

\section{2) Checking for any missing values:}

\section{\#Now check the missing values \\ miss=data.isnul||(|.any||) \\ miss\# Hence no missing values}

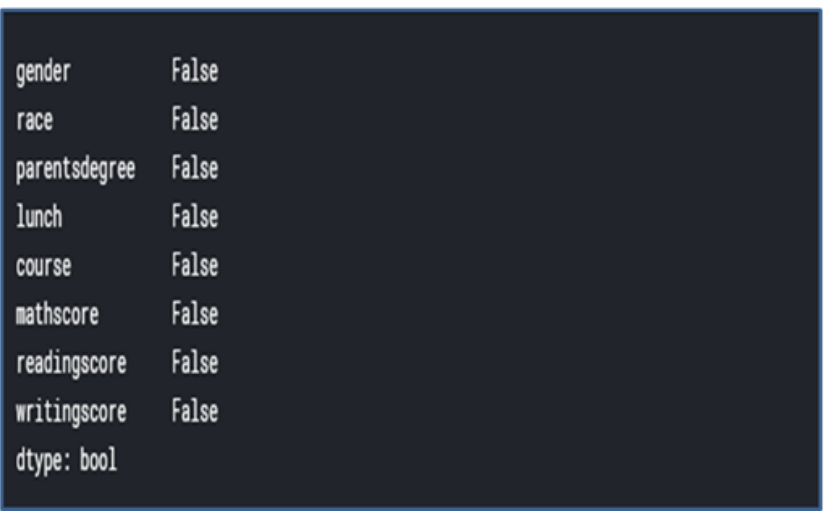

Figure12. Output after checking if any null values in the dataset

\subsection{Implementation}

For further implementation, an attribute called percentage is needed to represent the total scores of the students. For calculating the percentage, the mean of reading, writing and math score is considered. The mean is calculated as follows and the output is as in the Figure13.
\# Lets check the percentage

data['Percentage']=(data['mathscore']+data['readingscore']+data['writingscore'])/3 datahead()

\begin{tabular}{|c|c|c|c|c|c|c|c|c|c|}
\hline & gender & 1000 & paentisbegcee & lunen & couse & mathscove & reabingowe & untingsowe & Percentage \\
\hline 0 & femble & group $B$ & bacheol's degyee & senderio & none & 72 & 12 & 74 & 126060607 \\
\hline 11 & femble & group C & some colespe & senolarto & condeled & $\theta 9$ & 90 & 88 & 82333333 \\
\hline 21 & femble & group B & masters devged & standerio & none & 90 & 95 & 93 & 920000007 \\
\hline 31 & mate & grouph & assoodetes degeree & treeleducod & none & 47 & 57 & 4 & 49333333 \\
\hline 4 & $m b d$ & groupc & some college & standario & none & 76 & 78 & 75 & 76333333 \\
\hline
\end{tabular}

Figure13. Dataset after calculating percentage

\section{3) Dataframe.groupby()}

DataFrame.groupby (by=None, axis=0, level=None, as_index=True, sort=True, group _keys=True, squeeze=False, observed=False, ${ }^{* *}$ kwargs) data.groupby (['race', 'parentsdegree']).mean()

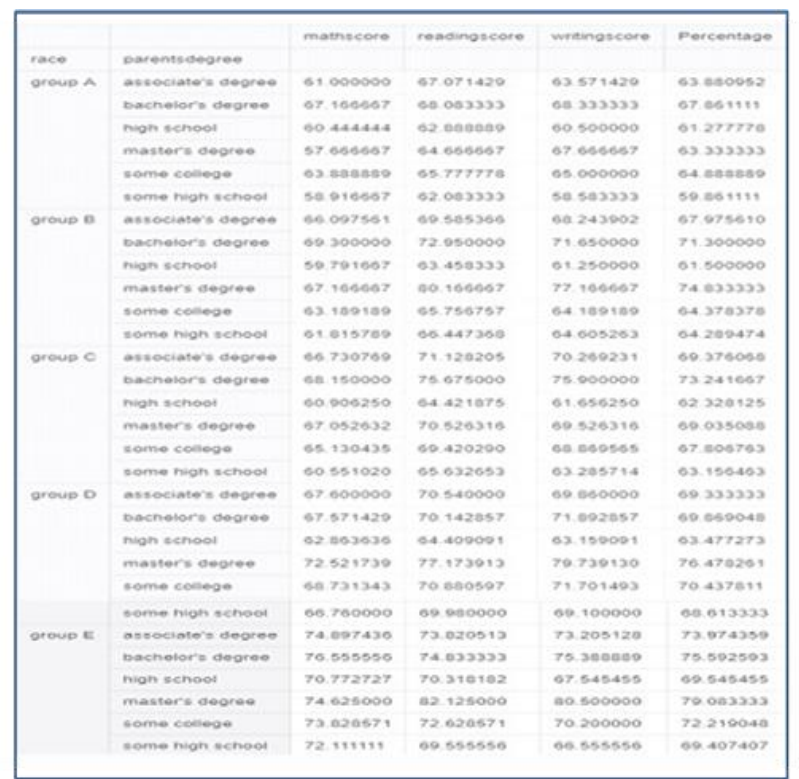

Figure14. Group-wise analysis

By the analysis of the above output in the Figure14, it can be observed that as race (Group) increases, the score also increases. This helps further in the prediction of at-risk students from the dataset.

Now checking the scores of the students by gender-based
Retrieval Number: C5440029320/2020@BEIESP
Published By:

Blue Eyes Intelligence Engineering

\& Sciences Publication

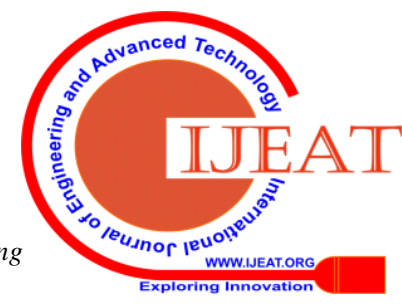




\section{NBC Model for Early Prediction of At-Risk Students in Course}

\# Lets check the score according to gender

data.groupby('gender').mean()

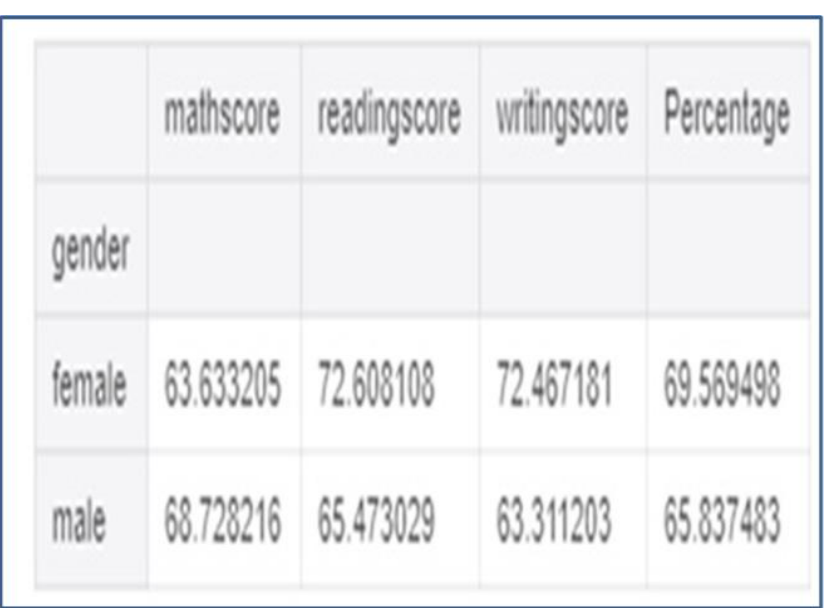

Figure15. Scores based on gender

The Figure15. clearly depicts that female percentage is greater than male percentage by some extent. While talking about marks, female math score is lower than the other two scores. The relation between the gender and the course completion is as shown in the Figure16.

\# Lets check the relationship between genders, course and percentage

course_gender=data.groupby(['gender','course']).mean().reset_index()

sns.factorplot $x=$ 'gender', $y=$ 'Percentage', hue='course', data=course_gender, kind='bar')

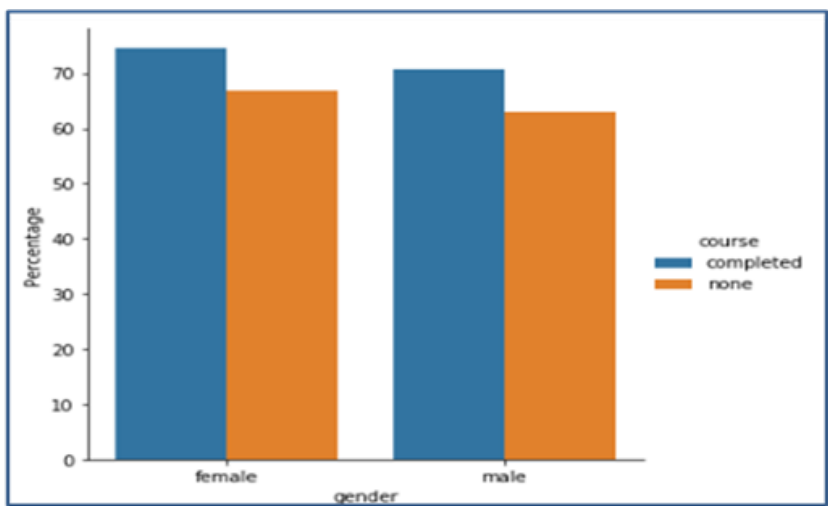

Figure16. Graph shows the relation between gender and course completion

\# Now we can say that Parents Degree is also crucial in students score

course gender=data.groupby(['gender','parentsdegree']).mean().reset index()

sns.factorplot(x='gender',y='Percentage', hue='parentsdegree',data=course_gender, kind='bar')

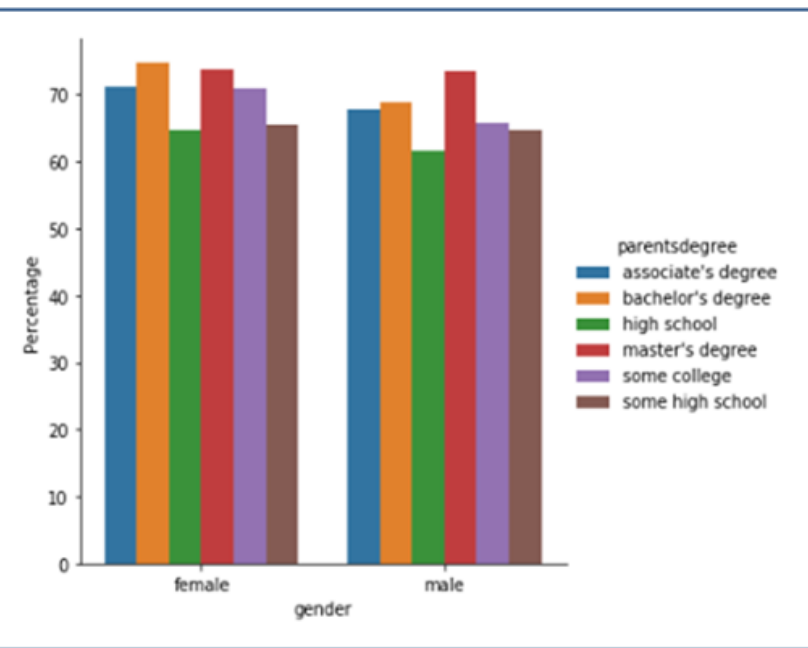

Figure17. Graph between gender and percentage with a parental degree as a factor

The students can be classified based on the parentsdegree.The "parentsdegree" column has 'has_degree' and 'no_degree' values. If a parentsdegree is either "high school" or "some high school" then, they comes under 'N0_Degree' category, else they come under 'has_Degree' category. The graph in the Figure17 shows the relation between gender and percentage with a parental degree as a factor.

\#Finding out different types of degree available data.parentsdegree.unique(

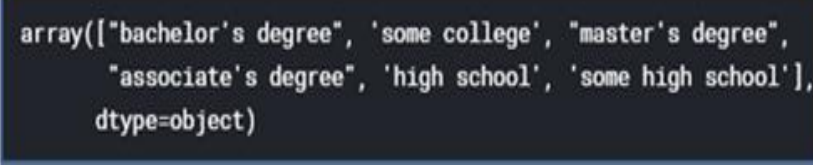

for $i$ in range(len(data)): if data.iloc [i,2] in ['high school','some high school'] data.iloc $[1,2]=$ No_Degree

else: data.iloc[i,2]='has_Degree'

data.head0

\begin{tabular}{|c|c|c|c|c|c|c|c|c|c|}
\hline & gender & race & parentisdegree & lunch & course & mathscove & reatingscove & urẗnoscoce & Percentiage \\
\hline & femde & group $B$ & has Degyed & standard & none & 72 & 72 & 74 & 7260000067 \\
\hline & femble & grovip C & has Degree & standard & completed & 69 & 90 & 88 & 82333333 \\
\hline & female & grovop $B$ & has Degree & standard & none & 90 & 95 & 93 & 9260000067 \\
\hline$\nu$ & male & group $A$ & has Degree & treeleduced & none & 47 & 57 & 44 & 49.3333333 \\
\hline 4 & male & group C & has Degree & standard & none & 76 & 78 & 75 & 76.333333 \\
\hline
\end{tabular}

Figure18. Output after changing parentsdegree

Published By:

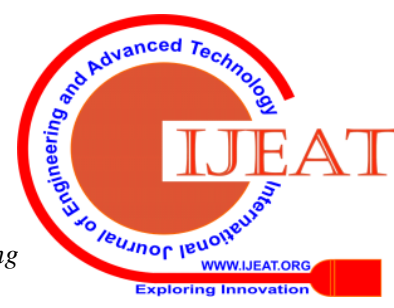


Now after changing the parentsdegree to either has_Degree or No_Degree, the students are again grouped.

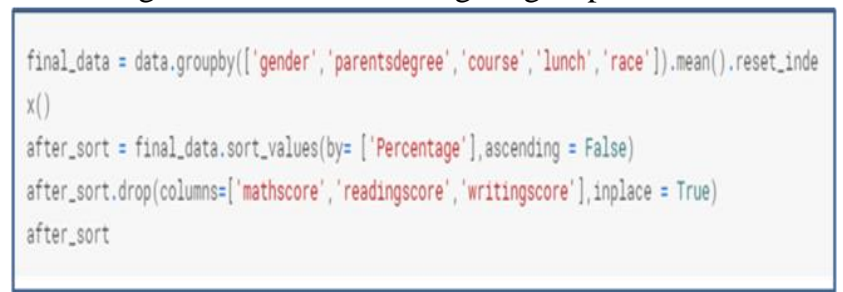

\begin{tabular}{|c|c|c|c|c|c|c|}
\hline & gender & parentsdegree & course & lunch & race & Percentage \\
\hline 28 & female & has_Degree & completed & standard & group $E$ & 84.023810 \\
\hline 5 & female & No_Degree & completed & standard & group A & 82.666667 \\
\hline 66 & male & has_Degree & completed & standard & group $C$ & 79.980392 \\
\hline 27 & female & has_Degree & completed & standard & group D & 79.738095 \\
\hline 65 & male & has_Degree & completed & standard & group $B$ & 79.100000 \\
\hline 26 & female & has_Degree & completed & standard & group C & 78.342593 \\
\hline 64 & male & has_Degree & completed & standard & group A & 77.761905 \\
\hline 8 & female & No_Degree & completed & standard & group D & 76.566667 \\
\hline 25 & female & has_Degree & completed & standard & group B & 76.555556 \\
\hline 23 & female & has_Degree & completed & free/reduced & group $E$ & 76.400000 \\
\hline 48 & male & No_Degree & completed & standard & group $E$ & 76.208333 \\
\hline 68 & male & has_Degree & completed & standard & group $E$ & 76.066667 \\
\hline 38 & female & has_Degree & none & standard & group $\mathrm{E}$ & 75.840580 \\
\hline 37 & female & has_Degree & none & standard & group D & 75.813725 \\
\hline 9 & female & No_Degree & completed & standard & group $E$ & 75.555556 \\
\hline 19 & female & No_Degree & none & standard & group $E$ & 75.2222222 \\
\hline 22 & female & has_Degree & completed & free/reduced & group D & 74.666667 \\
\hline 24 & female & has_Degree & completed & standard & group $A$ & 73.333333 \\
\hline 67 & maie & has_Degree & completed & standard & group D & 73.311111 \\
\hline 7 & female & No_Degree & completed & standard & group C & 72.333333 \\
\hline 63 & male & has_Degree & completed & free/reduced & group $E$ & 71.333333 \\
\hline 47 & male & No_Degree & completed & standard & group D & 71.300000 \\
\hline 20 & female & has_Degree & completed & free/reduced & group B & 71.250000 \\
\hline 36 & female & has_Degree & none & standard & group C & 70.809524 \\
\hline 43 & male & No_Degree & completed & free/reduced & group $E$ & 70.750000 \\
\hline 4 & female & No_Degree & completed & free/reduced & group $\mathrm{E}$ & 70.500000 \\
\hline 35 & female & has_Degree & none & standard & group $B$ & 70.466667 \\
\hline 62 & male & has_Degree & completed & free/reduced & group D & 70.000000 \\
\hline 60 & male & has_Degree & completed & free/reduced & group B & 69.904762 \\
\hline
\end{tabular}

\section{Figure19. Output after finding mean}

The Figure19. shows the generalization of all features.

\subsection{Result Analysis}

1. The result depicts that the Top students(mean) have completed their course, took standard Lunch, and they also had parent_Degree as a plus point.

2. Bottom students(mean) depicts that they didn't complete course, they didn't take good lunch, and their parent has no degree.

3. Out of Top 10(mean), 7 are female students

4. Interestingly, Out of Bottom 10(mean), 7 are male students

\#see top performers

print("Top 10 Performer $(n$ ",after_sort[:10])

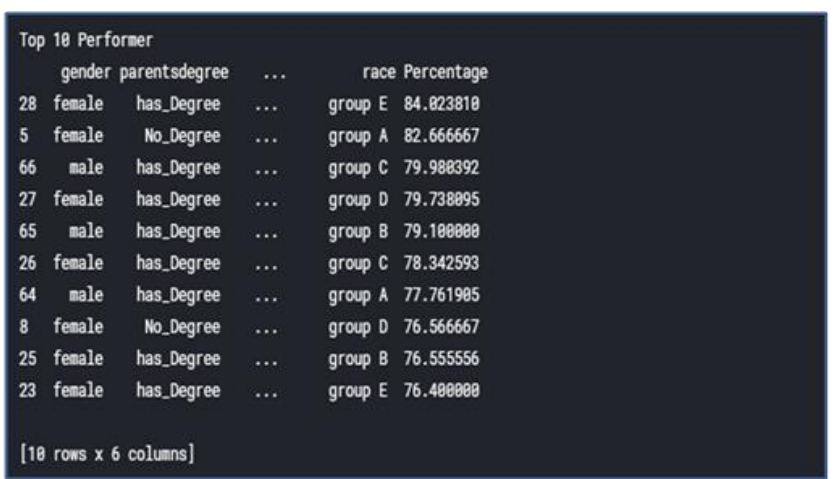

Figure20. Result of the top performers

After analyzing the results, it can be concluded that if a student completes the course, has standard lunch, then he can score good grades. The Top 10 performers are as shown in the Figure20 with Bottom Performers in Figure21.

\section{\#see bottom performers \\ print("Bottom Performer In",atter_sortf-10:])}

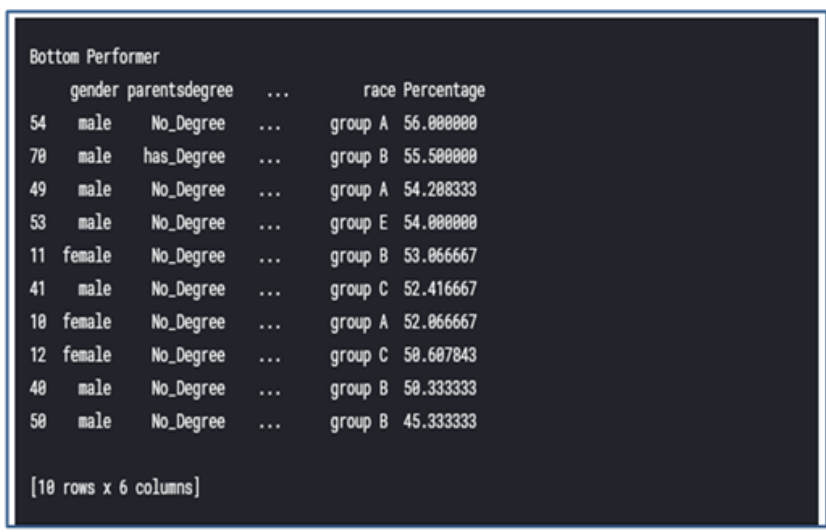

Figure21. Result of at-risk students

base = pd.get_dummies(final_data,columns=['gender','race','parentsdegree','course' 'lunch'], dtype =int)

base.sample()

base.info()

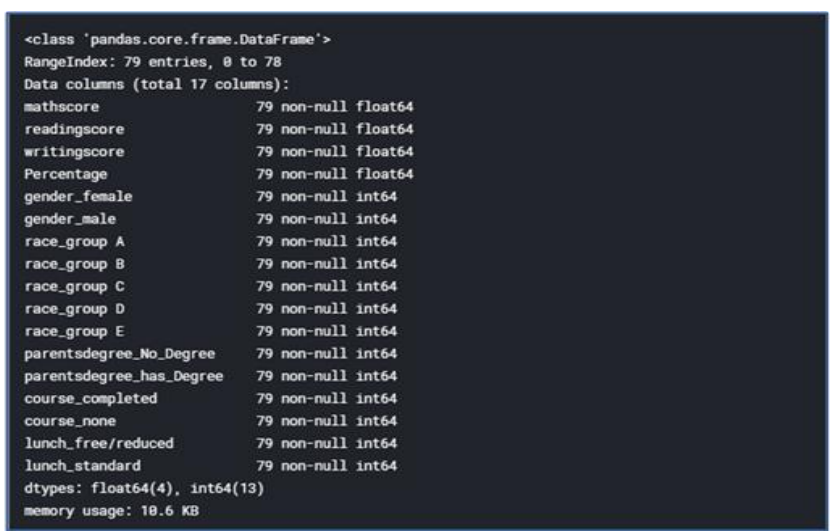

Figure22. Output of the base.info( ) command

Published By:

Blue Eyes Intelligence Engineering

Retrieval Number: C5440029320/2020@BEIESP

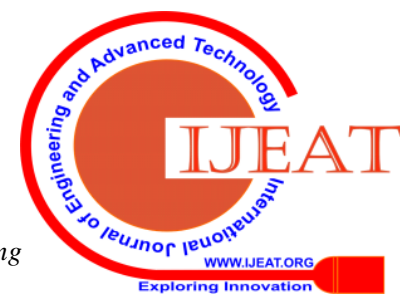




\section{NBC Model for Early Prediction of At-Risk Students in Course}

\section{CONCLUSION AND FUTURE ENHANCEMENTS}

The NBC model can be enhanced further by collecting more valid attributes in data set that helps in the prediction of the at-risk students. In this project, the most helping attributes are the parental education level and the student's reading score and writing score. More attributes like the student's percentage in the previous courses and present academic attendance help to increase the precision in the prediction. And the mean square error can be reduced by this.

\section{REFERENCES}

1. Machine learning for disease prediction by using neural networks, p. Sunanda, international journal of research and analytical reviews (ijrar), volume 6, Issue 2, Pages 712-719, Publisher, www.ijrar.org

2. http://www.ijrar.org/viewfull.php?\&p_id=IJRAR19K1534

3. A survey on machine learning: concept,algorithms and applications kajaree das1

4. bias-variance analysis of support vector machines for the development of svm-based ensemble methods - giorgio valentini

5. grade prediction with course and student specific models - agoritsa polyzou and george karypis

6. building course-specific regression-based models to identify at-risk students -mr. farshid marbouti

7. a deep feature selection approach - milad zafar nezhad

8. predicting at-risk students by analysing clicking behaviour in a virtual learning environment - annika wolff, zdenek zdrahal

9. survey on student performance prediction - mudit panwar

10. early detection of students at risk - predicting student dropouts using administrative student data and machine learning methods - johannes berens,

11. solutions to instability problems with sequential wrapper-based approaches to feature selection - kevin dunne

12. valentini, g., \& dietterich, t. g. (2004). bias-variance analysis of support vector machines for the development of svm-based ensemble methods.

13. carberry, a. r., siniawski, m. t., \& dionisio, j. d. n. (2012). standards-based grading:

14. links

15. https://en.wikipedia.org/wiki/machine learning

16. https://www.tutorialspoint.com/

17. https://www.expertsystem.com/machine-learning-definition/

18. https://towardsdatascience.com/applications-of-reinforcement-learnin g-in-real-world-1a94955bcd12

19. https://resources.interactions.com/machine-learning-important/

20. https://towardsdatascience.com/machine-learning-classifiers-a5cc4e1 b0623

21. https://www.edureka.co/blog/what-is-machine-learning/

22. https://bigdata-madesimple.com/8-ways-machine-learning-will-impro ve-education/

23. https://towardsdatascience.com/machine-learning-in-finance-why-wh at-how-d524a2357b56

24. https://theknowledgereview.com/machine-learning-impact-educationtechnology/

25. https://www.techopedia.com/how-machine-learning-can-improve-teac hing-excellence/2/33323

26. http://psycnet.apa.org/fulltext/1992-40647-001.html

27. https://www.analyticsvidhya.com/blog/2017/09/understaing-support-v ector-machine-example-code/

28. https://www.researchgate.net/publication/250145376_efficacy_of_par ticipating in_a first-year_seminar_on student_satisfaction_and rete ntion

29. https://www.mathworks.com/discovery/machine-learning.html

30. https://www.tandfonline.com/doi/abs/10.1080/07924360120043621

31. https://realpython.com/python-data-types/

32. 18.https://www.sciencedirect.com/science/article/pii/S03601 31516301634

\section{AUTHORS PROFILE}

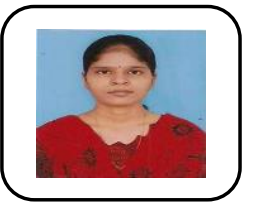

P. Sunanda, is Assistant Professor in the department of Computer Science \& Engineering a G.Pulla Reddy Engineering College, Kurnool India. She obtained her M.Tech degree in Computer Science from Jawaharlal Nehru Technological University, India and B.Tech degree in Computer Science and Engineering from Sri Krishna Devaraya University, India. Her current research interests are in the area of Computer Vision, Big data and machine learning.

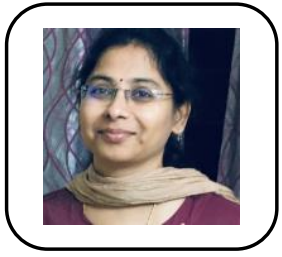

Dr. D. Kavitha, is a Professor in the department of Computer Science \& Engineering at G.Pulla Reddy Engineering College, Kurnool India. She received her Ph.D from Sri Krishna Devaraya University, India in 2012. She obtained her M.Tech degree in Computer Science from Jawaharlal Nehru Technological University, India and B.Tech degree in Computer Science and Engineering from Sri Krishna Devaraya University, India. Her current research interests are in the area of data analytics and machine learning. She published more than 30 papers in various International conferences and journals. 